\title{
Lymphomatosis cerebri: Multimodality imaging features and misdiagnosis analysis
}

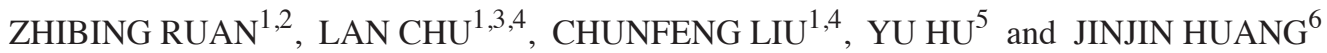 \\ ${ }^{1}$ Department of Neurology, The Second Affiliated Hospital of Soochow University, Suzhou, Jiangsu 215004; \\ Departments of ${ }^{2}$ Radiology and ${ }^{3}$ Neurology, The Affiliated Hospital of Guizhou Medical University, Guiyang, \\ Guizhou 550004; ${ }^{4}$ Institute of Neuroscience, Soochow University, Suzhou, Jiangsu 215004; ${ }^{5}$ School of Medical Imaging, \\ Guizhou Medical University; ${ }^{6}$ Department of Pathology, Guizhou Medical University, Guiyang, Guizhou 550004, P.R. China
}

Received March 2, 2021; Accepted July 15, 2021

DOI: 10.3892/ol.2021.12962

\begin{abstract}
Lymphomatosis cerebri (LC) is a significant challenge in terms of its clinical diagnosis due to it being a rare disease. The purpose of the present study was to investigate the multimodality imaging characteristics, clinical features and reasons for misdiagnosis of LC with the goal of potentially facilitating early and accurate diagnosis of this frequently misdiagnosed disease. In the present study, clinical data and cerebral multimodality imaging findings from 11 patients with LC proven based on pathology were retrospectively analyzed and reviewed with consultation of the literature. The results indicated that the common symptoms included cognitive decline (8/11), gait disturbance (9/11) and behavioral abnormalities (5/11). Cerebrospinal fluid analysis indicated that the number of cells and level of protein increased (8/10). All patients had both deep and lobar lesion distribution of bilateral cerebral white matter with equal or slightly low-density shadows on CT plain scan and slightly longer signals on T1and T2-weighted MRI. Most of the lesions (9/11) exhibited isointensity or slight hyperintensity on diffusion-weighted imaging and hyperintensity on apparent diffusion coefficient maps. In addition, five patients presented with a marked decrease in $\mathrm{N}$-acetylaspartate/creatine $(\mathrm{Cr})$ and increase in choline/Cr on ${ }^{1} \mathrm{H}$-magnetic resonance spectroscopy, including
\end{abstract}

Correspondence to: Dr Lan Chu, Department of Neurology, The Affiliated Hospital of Guizhou Medical University, 28 Guiyi Street, Guiyang, Guizhou 550004, P.R. China

E-mail: chulan8999@yeah.net

Abbreviations: LC, lymphomatosis cerebri; PCNSL, primary central nervous system lymphoma; FLAIR, fluid-attenuated inversion recovery; DWI, diffusion-weighted imaging; ADC, apparent diffusion coefficient; MRS, magnetic resonance spectroscopy; NAA, N-acetylaspartate; Cho, choline; $\mathrm{Cr}$, creatine; Lac, lactate; Lip, lipid; PET, positron emission tomography

Key words: lymphomatosis cerebri, primary central nervous system lymphoma, diffuse cerebral white matter lesions, multimodality imaging, misdiagnosis analysis an increase in lipid/Cr in 3 cases. Of these, one case exhibited no increase in lesion metabolism and 2 cases had slightly increased uptake on positron emission tomography/CT. The present study indicated that the multimodality imaging findings of LC have certain distinct characteristics and prompt recognition of these features may significantly improve early diagnosis and patient prognosis. Misdiagnosis may be mainly due to insufficient understanding knowledge of the condition and improper brain biopsy.

\section{Introduction}

Lymphomatosis cerebri (LC) is a rare variant of primary central nervous system lymphoma (PCNSL) (1-5) that is pathologically characterized by diffuse cerebral infiltration without a cohesive mass of malignant lymphoid cells around the blood vessels along the white matter fiber tracts. It mostly occurs in patients with normal immune function (2) and has a subacute onset, poor prognosis even with surgical treatment and a high recurrence rate, but it is sensitive to chemotherapy and radiotherapy $(2,6)$. Therefore, early diagnosis is significantly crucial for those patients, which mostly depends on imaging. However, the differentiated imaging features of LC have remained to be established in detail $(1-3,5,6)$.

The clinical and imaging findings of LC are significantly different from those of common PCNSL and are similar to those of central nervous system infection, inflammation, poisoning, metabolic disorders, demyelinating encephalopathy, autoimmune encephalitis and diffuse astrocytoma $(1,2,5,6)$, which easily leads to clinical misdiagnosis and unnecessary surgical treatment in certain cases. A study by Li et al (5) first indicated that perivascular curvilinear enhancement appears in diffuse infiltrative PCNSL, which is similar to observations made by our group.

According to the American Association of Neurological Surgeons, CT-guided stereotactic biopsy is a common procedure that allows neurosurgeons to diagnose a brain lesion. Performed in the operating room, the procedure involves the removal of a small piece of tissue, most commonly from the brain, but may include samples from the scalp, blood vessels or dura mater, and in most cases, the neurosurgeon uses stereotactic equipment to localize the preferable site for the 
biopsy (7). At present, early diagnosis of LC is challenging and misdiagnosis is not uncommon (1-3,5,8-10), but specific epidemiological data are lacking because it is a rare disease and most of the previous studies are case reports (1-6).

In order to investigate the multimodality imaging characteristics and clinical features of LC and the reasons for its misdiagnosis in the clinic, the clinical data and cerebral multimodality imaging findings from 11 patients with LC were retrospectively analyzed. The present study indicated that misdiagnosis mainly occurs due to limited knowledge of LC, since it is rare and seldom reported. Furthermore, corticosteroid treatment may cause the vanishing cancer phenomenon due to corticosteroid-induced apoptosis and lymphoma cell lysis in a short period of time $(4,5,11)$, which may result in false-negative pathology results of biopsies. However, no abnormal enhancement was observed in adjacent meninges or ependyma in any of those patients, which was not consistent with inflammatory lesions. Whether this sign has differential diagnostic value requires to be further studied and analyzed in a larger sample. In addition, CT-guided stereotactic biopsy for LC with extensive and diffuse lesions or posttreatment lesions has certain shortcomings because plain CT scans are not able to effectively distinguish active tumor areas from edema and necrotic areas, which may lead to false-negative pathology results if the amount of puncture specimen is limited. Inaccuracy of the puncture method, location or timing may be secondary reasons for misdiagnosis.

To the best of our knowledge, the present study was the first to summarize and discuss the clinical and multimodal imaging characteristics of LC by analyzing a relatively large number of cases, particularly in terms of the features of CTand MR-guided stereotactic biopsy, which is critical for the comprehensive understanding and early diagnosis of LC and will contribute to the reduction of misdiagnosis and avoid unnecessary surgery.

\section{Materials and methods}

Clinical data. The clinical data and cerebral multimodality imaging findings of 11 patients with LC (from November 2011 to December 2020) confirmed by pathology at The Affiliated Hospital of Guizhou Medical University (Guiyang, China), including those confirmed surgically $(n=5)$ and via CT-guided stereotactic biopsy $(n=6)$, were retrospectively reviewed. The extracted information mainly included clinical and histological data, cerebrospinal fluid findings, treatment variables and multimodality imaging findings [mainly including conventional $\mathrm{CT}$ and MR plain scan and contrast-enhanced scan, diffusion-weighted imaging (DWI), ${ }^{1} \mathrm{H}$-magnetic resonance spectroscopy (MRS) and ${ }^{18} \mathrm{~F}$-fluorodeoxyglucose (FDG)-positron emission tomography (PET)/CT scan]. All patients were diagnosed based on the following criteria: i) All patients presented with diffuse lesions of the bilateral cerebral white matter on imaging; ii) all patients were diagnosed with lymphoma by two experienced neuropathologists (each with 5 and 10 years of experience, respectively) according to $\mathrm{H} \& \mathrm{E}$ staining and immunohistochemical analysis; iii) All patients had complete multimodality imaging and clinical data. Patients with concurrent systemic lymphoma and intravascular lymphoma were excluded. All patients provided informed consent for the multimodality imaging examinations and for the use of personal data.

Radiological examination methodology. 128-slice CT scanning system (Toshiba Corporation) and MRI scans using unenhanced and multiphase enhanced techniques, $(n=11)$, DWI $(n=11),{ }^{1} \mathrm{H}-\mathrm{MRS}(\mathrm{n}=5)$ and ${ }^{18} \mathrm{~F}-\mathrm{FDG}-\mathrm{PET} / \mathrm{CT}(\mathrm{n}=3)$ were performed on patients with lymphomatosis cerebri.

The parameters were as follows: Voltage, $120 \mathrm{kV}$; current, $200 \mathrm{~mA}$; scan thickness, $5 \mathrm{~mm}$; interlayer spacing, $5 \mathrm{~mm}$; pitch, 0.5 ; and collimator, 16 slice $x 0.625 \mathrm{~mm}$. In order to make the multiple planar reconstruction, the scan for coronal, sagittal and other orientations were performed. A total of 60-100 ml of the nonionic iodine contrast agent iohexol (iodine $300 \mathrm{mg} / \mathrm{ml}$ ) were injected into the cubital vein at a rate of $3 \mathrm{ml} / \mathrm{sec}$ by using a high-pressure injector. The scan had 2 phase imaging: Arterial (delay, $30 \mathrm{sec}$ ) and venous (delay, $60 \mathrm{sec}$ ).

A Philips Achieva 3.0-T whole-body MRI system (Philips Healthcare) and head phased array coil (an 8-channel phased array coil) were used. Before the scan, all patients fasted 4-8 h. T2-weighted fluid-attenuated inversion recovery (FLAIR), T1-weighted images (T1WI) and T2-weighted images (T2WI) were collected as baseline MRI data. After intravenous administration of $0.1 \mathrm{mmol} / \mathrm{kg}$ gadopentetate dimeglumine (Beilu Inc.), all patients took the postcontrast T1WI. DWI was performed by using B-values of $0 \mathrm{sec} / \mathrm{mm}^{2}$ and $1,000 \mathrm{sec} / \mathrm{mm}^{2}$. The mean apparent diffusion coefficient (ADC) values in both the lesions and normal-appearing white matter were measured by two experienced neuroradiologists (each with 5 and 10 years of experience). MR spectroscopy was performed with a long echo time (135 msec) as a multivoxel 2D exam encompassing the lesion. Major metabolites were detected, such as choline (Cho), N-acetylaspartate (NAA), creatine (Cr), lipids (Lip), and lactate (Lac) peaks. In order to quantify other metabolites for each spectrum, the peak height of total creatine $(\mathrm{Cr})$ was used as the internal reference.

A Philips GEMINI TF 64 PET/CT machine (Philips Healthcare) with ${ }^{18} \mathrm{~F}-\mathrm{FDG}$ (purity, $>95 \%$ ) was performed. Before the scan, patients were requested to fast for $6 \mathrm{~h}$ and the fingertip fasting blood glucose level needed to be $<11 \mathrm{mmol} / 1$ (normal range, 3.9-6.1 mmol/l). After ${ }^{18} \mathrm{~F}-\mathrm{FDG}$ (3.7 MBq/kg body weight) was injected into the cubital vein, the scan was performed in the supine position for $1 \mathrm{~h}$ in a quiet dark room. Parameters for CT were as follows: Voltage $=120 \mathrm{kV}$; current $=120 \mathrm{~mA}$; CT reconstruction thickness $=5 \mathrm{~mm}$; and interval $=5.0 \mathrm{~mm}$. CT data were used to attenuate and correct the PET images. Regarding PET image reconstruction, ordered subset iterative expectation maximization for was performed with a thickness of $5 \mathrm{~mm}$ and an interval distance of $5 \mathrm{~mm}$. In order to get cross-sectional PET, sagittal PET, coronal PET, CT and PET/CT fusion images, reconstructed images of CT and PET were transferred to a dedicated workstation and analyzed using IntelliSpace Portal v.6.5 (Philips Healthcare). Head lesions were outlined as the region of interest using a semiquantitative method to measure the maximum standardized uptake value (SUVmax) of radioactivity.

Analysis of the multimodal imaging data. Imaging data were reviewed independently by two expert radiologists (with 10 and 15 years of experience) using a double-blind 
method and any disagreements were resolved by consensus. All scans were reviewed with a focus on the observation of the lesion location in the brain, patterns of contrast enhancement, DWI signal and the corresponding ADC map, MRS metabolic patterns and the SUVmax of radioactivity on PET/CT. The lesion distribution included the deep cerebral white matter, cortical and subcortical white matter, basal ganglia and the thalamus, subtentorial cerebellum and brainstem. Contrast enhancement was defined as patchy when a minimally or moderately heterogeneous, poorly defined area of contrast enhancement was present, regardless of the size. The imaging analysis and diagnosis of each patient were compared with the pathological findings.

$H \& E$ staining. After the sections $(5 \mu \mathrm{m})$ were deparaffinized in xylene and rehydrated with an alcohol gradient, they were stained with hematoxylin solution and differentiated in $1 \%$ hydrochloric alcohol, followed by dehydration in $95 \%$ ethanol. The sections were counterstained with $1 \%$ eosin solution.

Immunohistochemical analysis. Paraffin sections $(5 \mu \mathrm{m})$ were deparaffinized in xylene, rehydrated with an alcohol gradient and processed for antigen retrieval by boiling in $10 \mathrm{mM}$ citrate buffer ( $\mathrm{pH}$ 6.0) for 2-3 min. The sections were incubated in $1 \% \mathrm{H}_{2} \mathrm{O}_{2}$ in PBS for $15 \mathrm{~min}$ at room temperature to quench endogenous peroxidase. To block nonspecific binding, sections were incubated in $3 \%$ bovine serum albumin (Wuhan Boster Biological Technology, Ltd.) for $10 \mathrm{~min}$. Sections were then incubated with anti-CD20 (cat. no. OTI4B4) and anti-CD3 (cat. no. OTI3E10; both used at 1:150 dilution; OriGene Technologies, Inc.) overnight at room temperature. After washing, antibodies were visualized using the HRP-polymer kit (Wuhan Boster Biological Technology) with incubation for 15-20 $\mathrm{min}$ at room temperature, followed by processing with 3,3'-diaminobenzidine tetrahydrochloride as the chromogen for 1-3 min at room temperature.

\section{Results}

Clinical features. The present case series consisted of 7 males and 4 females with an age ranging from 28 to 78 years (average age, $53.2 \pm 13.3$ years; median age, 56 years). The common symptoms included cognitive decline $(\mathrm{n}=2,72.73 \%)$, gait disturbance $(n=2,81.82 \%)$ and behavioral disturbance $(n=2$, $45.45 \%$ ) with subacute onset (Table I). Examination of the cerebrospinal fluid indicated that the number of cells and the protein level increased $(\mathrm{n}=8,80 \%)$, while the sugar content $(n=2,20 \%)$ and chloride levels decreased $(n=4,40 \%)$. A total of five patients were treated with surgery and the remaining six patients were subjected to CT-guided stereotactic biopsy. Among the patients, 5 cases were misdiagnosed as having inflammatory lesions, 3 as metabolic or toxic lesions and 3 as diffuse astrocytoma based on clinic symptoms and imaging results. The general and clinical information of these patients is summarized in Table I.

Multi-modality imaging features. All patients had both extensive deep and lobar cerebral lesion distribution in the bilateral cerebral white matter (images of representative cases in Figs. 1-4), which involved the cerebral cortex and

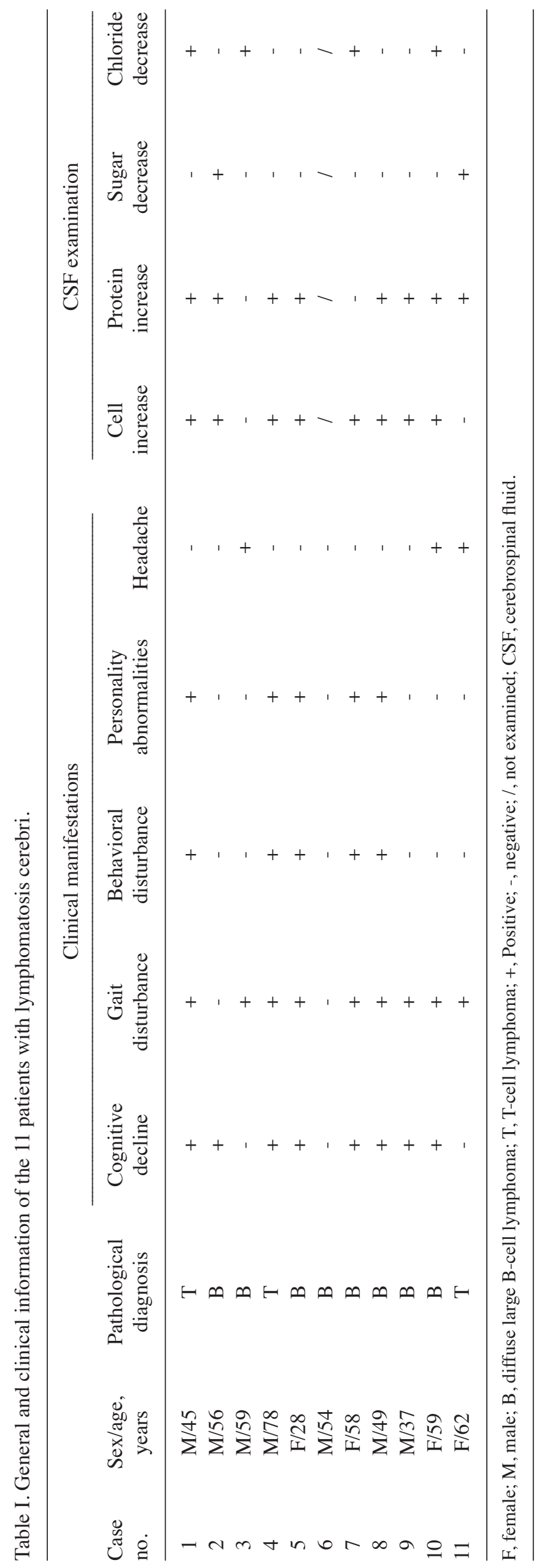


A

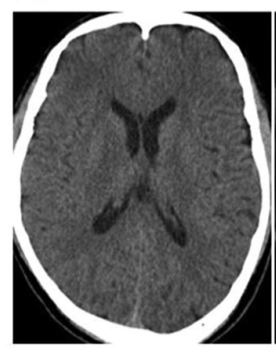

G

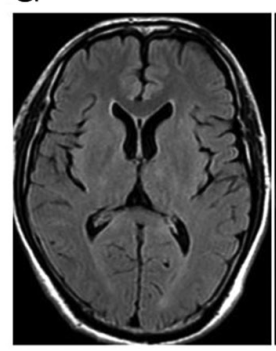

M

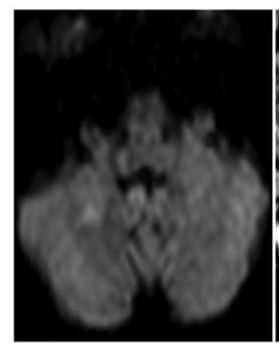

B

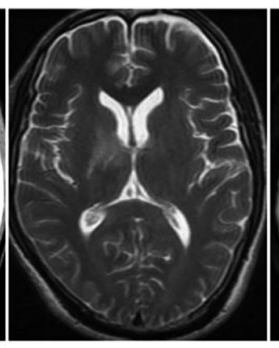

C

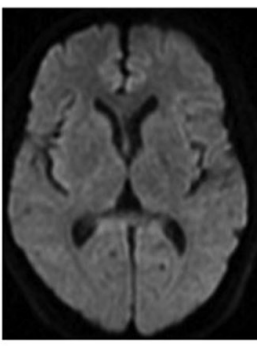

D

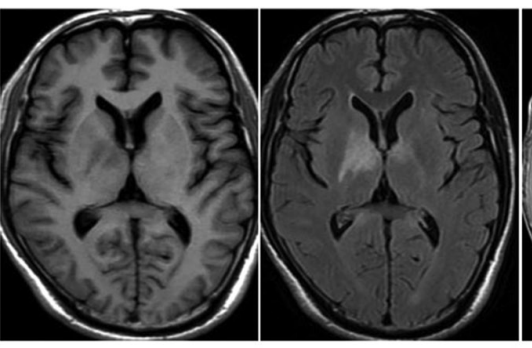

$\mathrm{F}$

$\mathrm{H}$

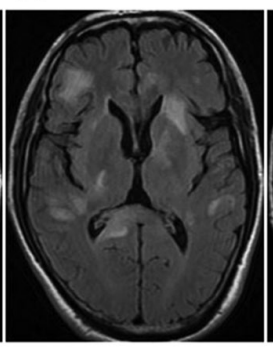

I

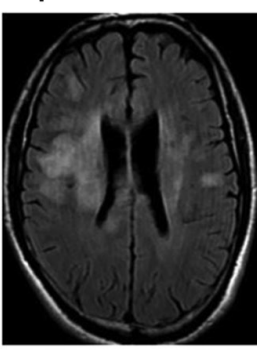

$\mathrm{J}$

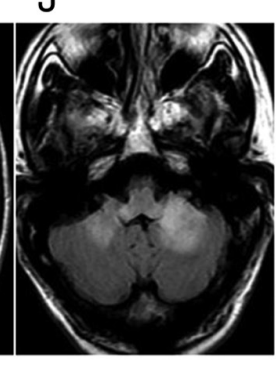

$\mathrm{K}$

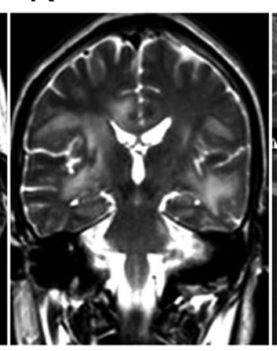

L
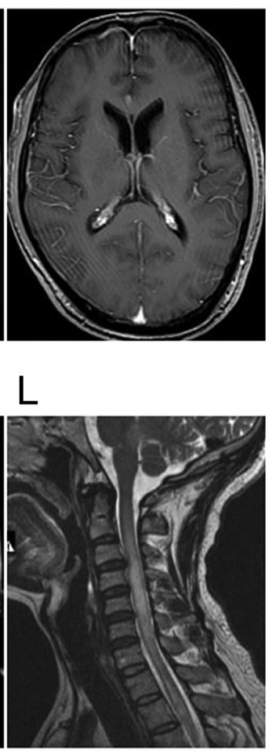

$\mathrm{N} \quad \mathrm{O}$

$\mathrm{P}$

Q
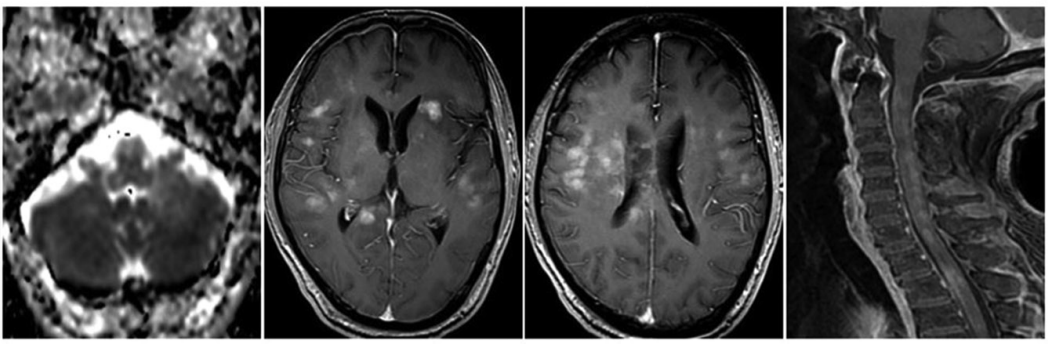

Figure 1. A 45-year-old male with diffuse T-cell lymphomatosis cerebri presented with memory loss, fatigue, lethargy and slow response for 4 months, which had been aggravated for 10 days. (A-F) Initial examination: (A) Plain axial CT scan indicated no obvious abnormalities. MRI: Patchy bilateral basal ganglia signals with slightly longer signals on (B) T1WI and (C) T2WI, and (D) high FLAIR signal. (E) DWI exhibited no diffusion limitation and (F) the T1WI enhanced scan revealed no enhancement. Re examination after 1 month of cortisol treatment: (G) FLAIR sequence indicated that the original abnormal patchy bilateral basal ganglia signals were significantly less pronounced on re-examination. Symptom recurrence re-examination after 2 months: (H-J) Axial FLAIR sequence indicated that the bilateral basal ganglia area had slightly higher signals than at the initial examination and the frontal lobe, temporal lobe, parietal lobe, radiation coronal area, corpus callosum and bilateral cerebellar hemispheres had new, similar lesions along the line; (K) the corticospinal tract was observed to infiltrate the cerebral feet and pons. (L) Sagittal T2WI revealed new, similar lesions in the cervical and upper thoracic spinal cord. (M) Cerebellar and brainstem lesions on DWI produced equal or slightly high signals and (N) apparent diffusion coefficient images displayed mainly slightly high signals (O-Q) T1WI enhanced scan indicated that the abovementioned brain lesions were patchy and nodular with obvious enhancement. FLAIR, fluid-attenuated inversion recovery; T1WI, T1-weighted imaging; DWI, diffusion-weighted imaging.

subcortical white matter in 8 cases $(72.72 \%)$, basal ganglia in 7 cases $(63.64 \%)$, the thalamus in 5 cases $(45.45 \%)$, cerebellum in 6 cases $(54.54 \%)$ and the brainstem in 6 cases $(54.54 \%)$. A total of six patients underwent spinal cord examination and 1 patient $(16.67 \%)$ presented with cervical spinal cord and thoracic spinal cord infiltration (Fig. 1L and Q). The lesions were mostly limited and asymmetrical in the early stage and the number and range of lesions increased along with disease progression (Figs. 1 and 2). However, the lesions exhibited significant improvement in the short term after treatment with high-dose corticosteroid pulse therapy (Fig. 2H) but exhibit recurrence and progressive aggravation in the long term (Fig. 1H-L). Plain CT scans exhibited equal or slight low-density shadows (Figs. $1 \mathrm{~A}$ and $3 \mathrm{G}$ ) and 4 cases (36.36\%) had isodensity and correct diagnoses were missed. MRI displayed slightly longer T1WI and T2WI signals in all patients $(\mathrm{n}=11,100 \%)$ (Figs. 1-4). At the initial visit, a lack of contrast enhancement and subtle patchy enhancement patterns were observed in 6 patients $(n=6,54.55 \%)$ (Figs. $1 F$ and 2D) and 5 patients $(\mathrm{n}=5,45.45 \%)$ (Fig. 3D-F), respectively, and the follow-up imaging in the 5 patients who lacked enhancement indicated obvious enhancement of multiple patches and nodules or masses $(n=5,45.45 \%)$ (Figs. $2 \mathrm{G}$ and $4 \mathrm{C})$. Most of the lesions $(n=9,81.82 \%)$ displayed with isointensity or slight hyperintensity compared to normal brain tissue on DWI and hyperintensity on ADC maps (Figs. 1E and 2D). Follow-up observations gradually indicated extensive diffusion limitation (Figs. 2F and 4B). A total of five patients ( $\mathrm{n}=5,100 \%)$ presented with marked decreases in NAA/Cr and increases in $\mathrm{Cho} / \mathrm{Cr}$ and $\mathrm{Lip} / \mathrm{Cr}(\mathrm{n}=3,60 \%)$ on ${ }^{1} \mathrm{H}-\mathrm{MRS}$. Among the three patients who underwent ${ }^{18} \mathrm{~F}-\mathrm{FDG}$ PET/CT examination, 1 (33.33\%) exhibited no abnormal increase in lesion metabolism, while $2(66.67 \%)$ had slightly increased tumor metabolism (Fig. 3G-I). The multimodality imaging findings of the patients are summarized in Table II.

Pathological findings. A total of eight patients were diagnosed with diffuse large B-cell lymphoma and 3 patients 


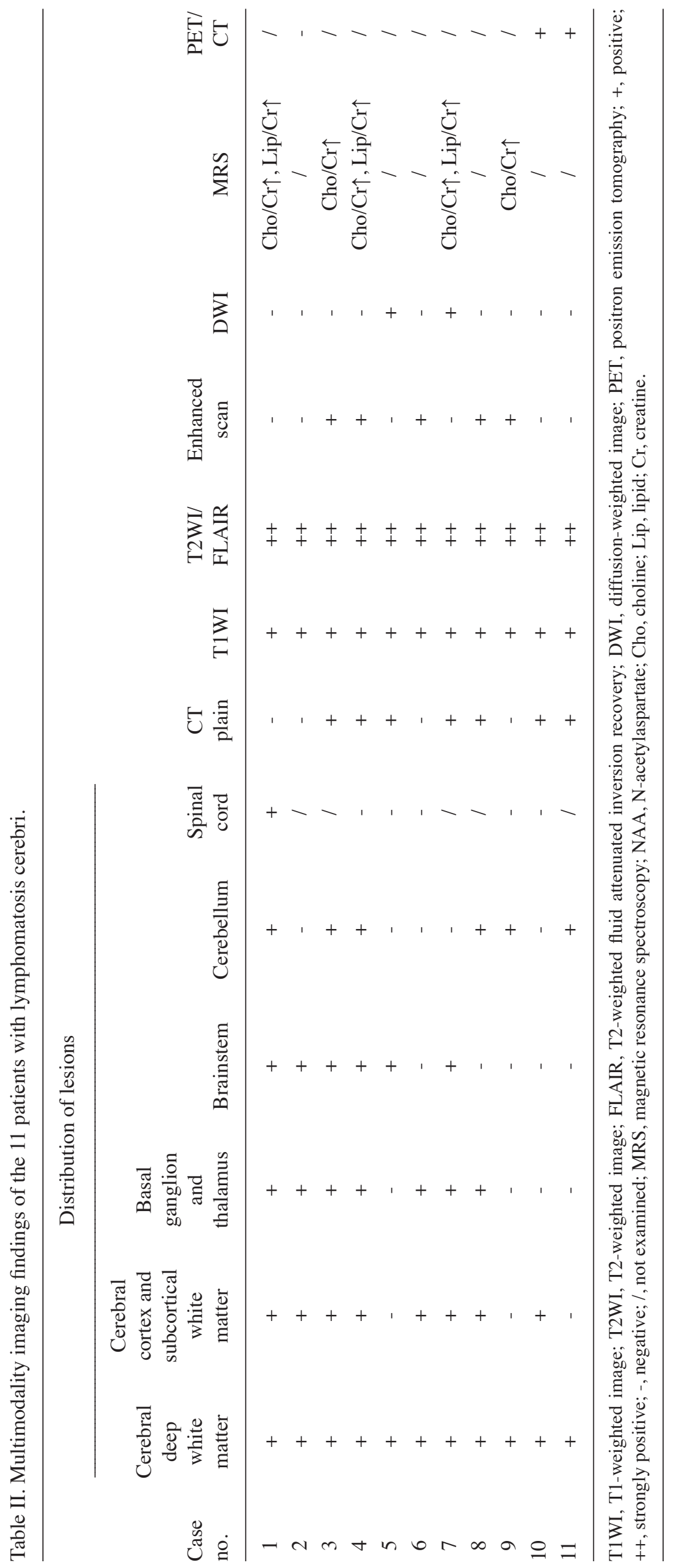



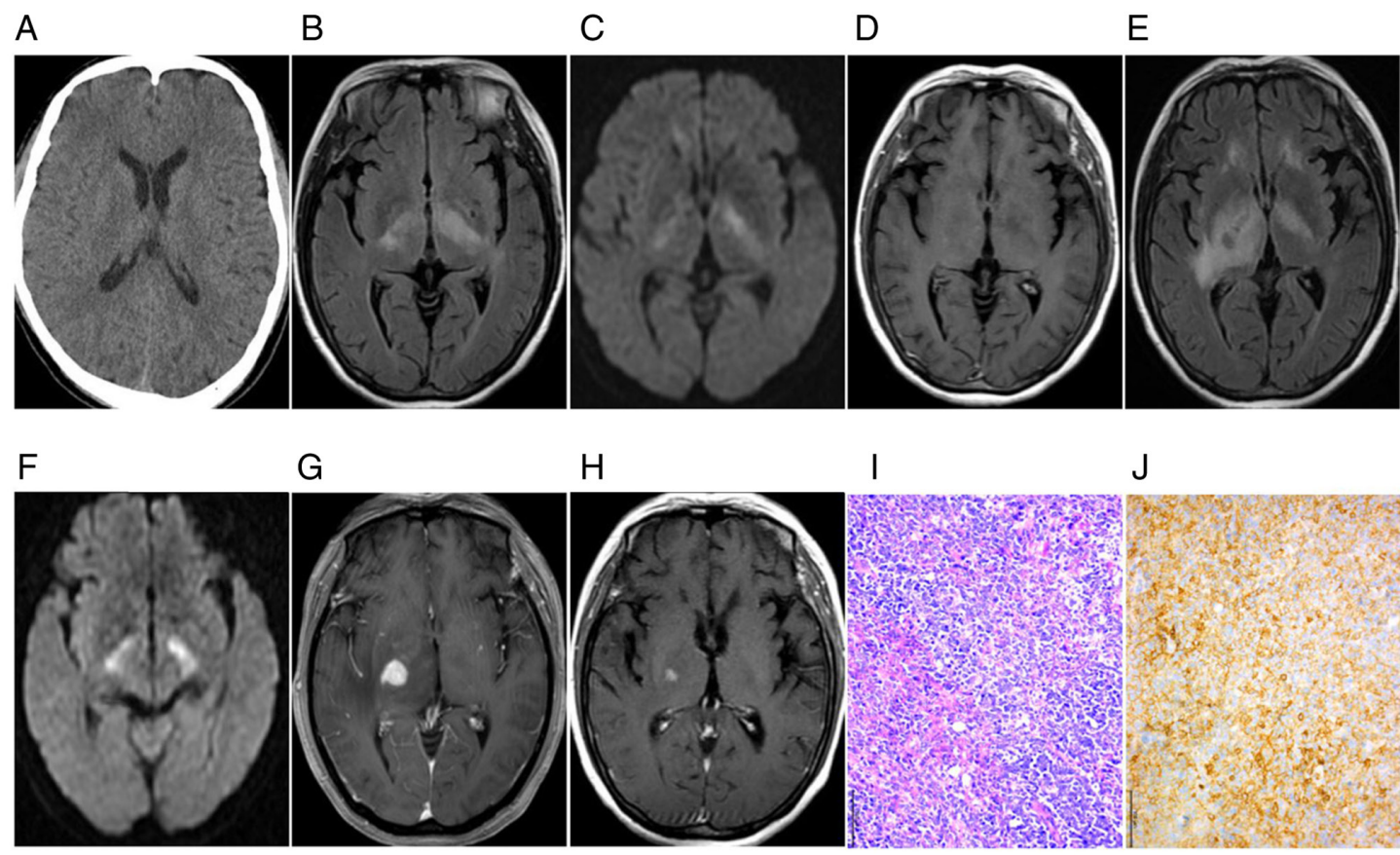

Figure 2. A 58-year-old female with diffuse large B-cell lymphomatosis cerebri presented with unstable gait on both lower limbs with memory loss and personality changes for 1 month. (A) Axial T2WI image revealed bilateral basal ganglia patches with slightly longer T2 signal lesions. (B) FLAIR image with a slightly higher signal and clearer display, and certain lesions exhibited limited spread and (C) a slightly high signal on DWI. (D) T1WI enhanced scan revealed no obvious abnormal enhancement. Disease progression was reviewed 4 months later. (E) In the axial FLAIR image, the bilateral basal ganglia area had flaky hyperintensity and (F) on DWI, more restricted diffusion than at earlier time points was observed. (G) The T1WI enhanced scan revealed small patchy and nodular enhancement. Re-examination after 2 months of cortisol treatment: $(\mathrm{H}) \mathrm{T} 1 \mathrm{WI}$ enhanced scan indicated that the original lesions were significantly reduced compared with the previous time-points and the enhancement range and degree were significantly reduced. (I) H\&E staining showed that the brain specimen with large- to medium-sized lymphoid cells diffusely infiltrated along the white-matter tracts without mass formation with visible immunoblasts and central blasts. (J) Immunohistochemistry revealed that the atypical cells were positive for CD20, a marker for B cells (magnification, x200). FLAIR, fluid-attenuated inversion recovery; T1WI, T1-weighted imaging; DWI, diffusion-weighted imaging.

were diagnosed with T-cell lymphoma. Microscopically, high proliferation of large lymphoid cells with perivascular infiltration and high mitotic index were observed. Tumor cells were permeated by reactive small $\mathrm{T}$ and $\mathrm{B}$ lymphocytes, macrophages, activated microglial cells and reactive astrocytes (Figs. 2I and 4D). Of note, lymphoid cells in all those patient sections expressed CD20 (11/11) (Fig. 2J). In sections from certain patients (6/11), a small number of reactive $\mathrm{CD}^{+} \mathrm{T}$ cells were present in the vicinity of neoplastic B cells (Fig. 4E).

\section{Discussion}

LC predominantly occurs in middle-aged and elderly males. The common clinical manifestations include progressive cognitive dysfunction and gait instability, as well as behavioral abnormalities and personality changes $(1,5,7,9,11)$. In the present study, 8 cases were large B-cell lymphoma and 3 cases were T-cell lymphoma. Immunohistochemistry analysis indicated that these lymphoid cells expressed CD20 in the majority of the cases and a small number of reactive $\mathrm{CD}^{+} \mathrm{T}$ cells were observed in the vicinity of neoplastic B cells, in line with the previous literature (4,7-10).

Most of the cases of the present study were supratentorial with bilateral onset and the most common lesion location was the deep white matter of the bilateral cerebral hemispheres. The lesions were mostly localized and asymmetric in the early stage, increasing in size as the disease progressed. They also tended to be bilaterally symmetric, with a propensity to infiltrate the entire central nervous system along the corticospinal tract $(5,12)$. The distribution and development characteristics of the lesions were consistent with the biological properties of lymphoma cells, which have a tendency to spread along the white matter fiber tracts $(5,13)$, supporting the theory that the disease is a whole-brain disease (13). Therefore, the progressive development of LC suggests the importance of dynamic clinical imaging observations in earlier diagnosis of this disease $(14,15)$.

Most cases of the present study had no enhancement at the early stage but then presented with enhancement at follow-up and the enhancement mode changed as the lesions progressed, similar to previous findings $(5,7,10,11,13,16)$. This observation is related to the progressive pathological features of lymphoma cells diffusely infiltrating the brain tissue surrounding the blood vessels. Histopathological analysis indicated infiltration of tumor cells and destruction of microvessels by small round lymphocytes, providing supportive evidence of blood-brain barrier disruption with the appearance of enhancement on imaging.

In addition, most of the patients in this group (81.82\%) exhibited no limited diffusion on the initial DWI but follow-up observation gradually indicated extensive limited diffusion, which was consistent with previous reports $(2,7,9)$. It may be speculated that this was a result of the cell density in the tumor area continuing to increase and the intercellular space becoming narrower as the disease progressed, eventually leading to restricted local water molecule diffusion. While 
A

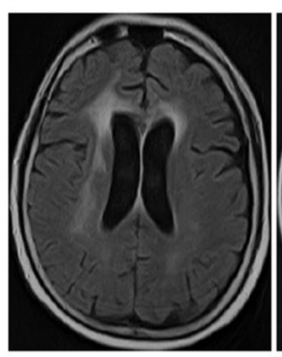

B

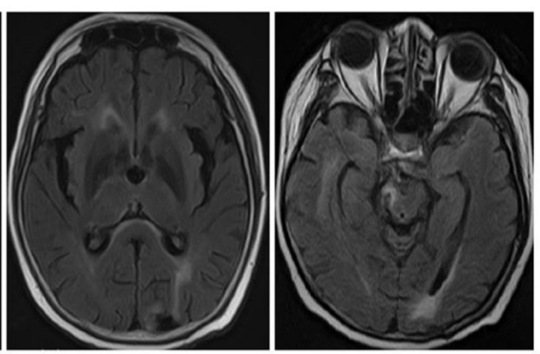

D

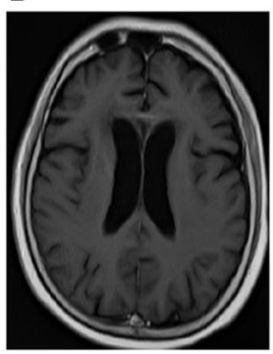

$\mathrm{E}$

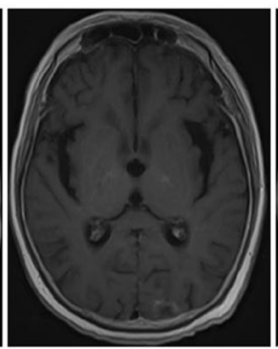

$\mathrm{G}$

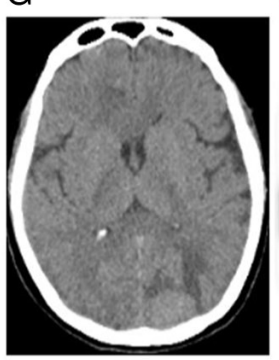

$\mathrm{H}$

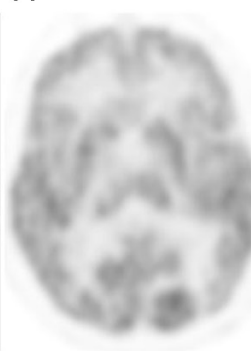

$\mathrm{F}$

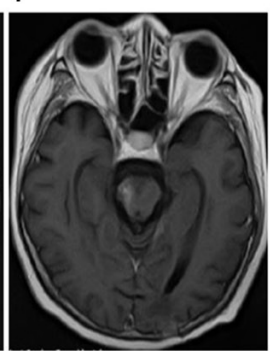

I

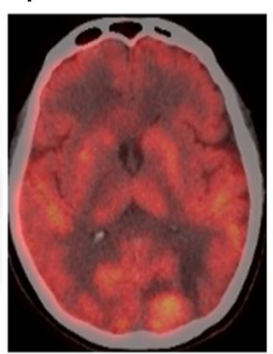

Figure 3. A 59-year-old male with diffuse large B-cell lymphomatosis cerebri presented with headache and bilateral lower extremity weakness with walking instability for 14 days. (A-C) Axial fluid-attenuated inversion recovery images revealed small patchy and equal slightly hyperintensive lesions in the bilateral basal ganglia, temporo-occipital areas, genu of the corpus callosum and pons. (D-F) T1-weighted imaging enhanced scan displayed the abovementioned lesions with small spot-like enhancement. (G) Positron emission tomography/CT indicated that the partial lesions were equal or had slightly low-density shadows. (H) ${ }^{18} \mathrm{~F}$-fluorodeoxyglucose was slightly concentrated and (I) slightly high metabolism was indicated in PET.

three patients underwent ${ }^{18} \mathrm{~F}-\mathrm{FDG}-\mathrm{PET} / \mathrm{CT}$ examination, it remained challenging to differentiate between early negative $\mathrm{PET} / \mathrm{CT}$ results, non-neoplastic lesions such as inflammation and late high uptake from other malignant lesions. At present, the characteristics of LC-related PET/CT findings are poorly understood and the characteristic findings require to be summarized in larger samples.

In summary, despite the small sample size of the cohort, the present results still indicated that the clinical manifestations and multimodal imaging findings of LC have certain characteristic features, including extensive diffuse bilateral cerebral white matter lesions, particularly in deep and lobar cerebral regions, with a tendency to infiltrate the entire central nervous system along the corticospinal tract, along with no obvious diffusion limitation on DWI and no obvious enhancement or with varied enhancement patterns as the lesions progressed. Prompt recognition of these features by clinicians and radiologists is important for the early diagnosis and improved prognosis of LC. Apart from that, stereotactic biopsy guided by MRI or enhanced CT is another ideal choice $(7,17)$, while

A

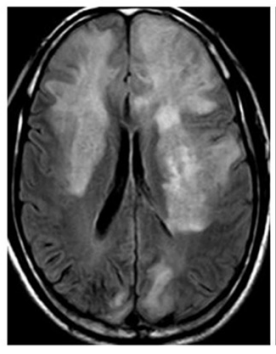

B

D

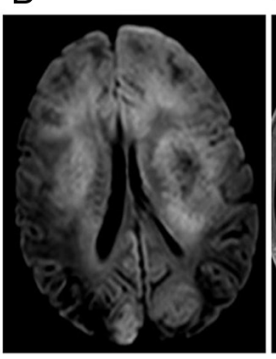

C

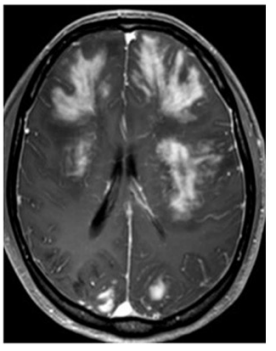

$\mathrm{E}$

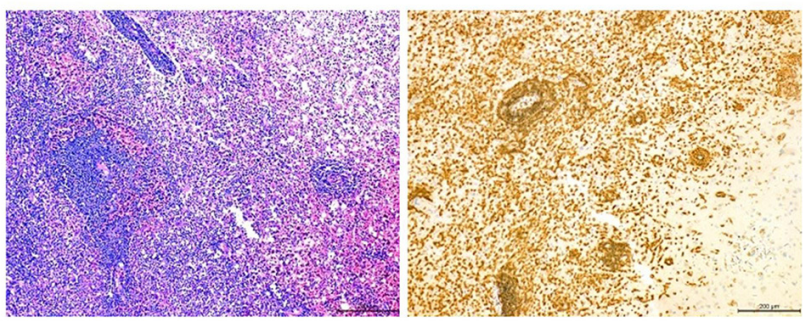

Figure 4. A 78-year-old male with diffuse large T-cell lymphomatosis cerebri presented with unresponsiveness for 1 year, weak right limb for 1 month and abnormal behavior for 10 days. (A) Axial fluid-attenuated inversion recovery image indicated diffuse patchy and large patchy hyperintensities throughout both cerebral hemispheres (mainly deep brain white matter) (B) Diffusion-weighted imaging revealed extensive and uneven hyperintensity in the lesions and the corresponding apparent diffusion coefficient maps (not shown) indicated water restriction. (C) T1-weighted imaging enhanced scan featured patchy and nodular enhancement. (D) H\&E staining showed brain biopsy specimen with small to medium lymphoid cells that grew with a nodular or fuzzy nodular pattern (magnification, x100; scale bar, $200 \mu \mathrm{m}$ ). (E) Immunohistochemistry revealed that the atypical cells were positive for CD3, a marker for T cells (magnification, x100; scale bar, $200 \mu \mathrm{m}$ ).

puncturing biopsy should be avoided after corticosteroid treatment.

\section{Acknowledgements}

Not applicable.

\section{Funding}

This research was supported by grants from the Science and Technology Program of Guizhou Province [platform talents (2018)5779-72 and LC (2017) no. 7206].

\section{Availability of data and materials}

The datasets used and/or analyzed during the current study are available from the corresponding author upon reasonable request.

\section{Authors' contributions}

ZR, LC and YH contributed to the extraction and review of patient data from the clinical records and images, as well as the conceptualization of the study and writing of the manuscript. LC and CL interpreted the patients' clinical data. YH performed the analysis for the DWI and MRS parts and wrote part of the manuscript and edited it. JH interpreted the histological examination results of the specimens. ZR and LC 
confirmed the authenticity of all the raw data. All authors read and approved the final manuscript.

\section{Ethics approval and consent to participate}

The present study was approved by the Ethics Committee of the Affiliated Hospital of Guizhou Medical University (Guizhou, China) and was performed according to the Declaration of Helsinki guidelines. Written informed consent was obtained from all participants for inclusion in the study.

\section{Patient consent for publication}

Written informed consent was obtained from all participants for publication of their images in this manuscript.

\section{Competing interests}

The authors declare that they have no competing interests.

\section{References}

1. Hatanpaa KJ, Fuda F, Koduru P, Young K, Lega B and Chen W: Lymphomatosis cerebri: A diagnostic challenge. JAMA Neurol 72: 1066-1067, 2015.

2. Tao J, Zhang Y, Huang Z, Li H and Wang L: A case of lymphomatosis cerebral with the features characterized by bilateral cerebral diffuse white matter lesions. Chin J Nerv Ment Dis 45: 53-56, 2019.

3. Yamaura G, Ogasawara A, Ito T, Ohsugi S, Kanatsuka Y, Hayashi R, Iwashita H, Hayashi H, Koyano S, Yamaguchi S and Tanaka F: Pathologically proven gadolinium-enhanced MRI lesions in the bilateral corticospinal tracts in lymphomatosis cerebri. Intern Med 59: 2931-2934, 2020.

4. Gametchu B: Glucocorticoid receptor-like antigen in lymphoma cell membranes: Correlation to cell lysis. Science 236: 456-461, 1987.

5. Li L, Rong JH and Feng J: Neuroradiological features of lymphomatosis cerebri: A systematic review of the English literature with a new case report. Oncol Lett 16: 1463-1474, 2018.

6. Duan L, Wang X, Zhang M and Wu J: Clinical analysis of 75 cases of primary central nervous system lymphoma. Chin J Oncol 45: 88-91, 2018.
7. American Association of Neurological Surgeons: Available from: https://www.aans.org/en/Patients/Neurosurgical-Conditions-andTreatments/Stereotactic-Brain-Biopsy. Accessed May 11, 2021.

8. Yu H, Gao B, Liu J, Yu YC, Shiroishi MS, Huang MM, Yang WX and Guan ZZ: Lymphomatosis cerebri: A rare variant of primary central nervous system lymphoma and MR imaging features. Cancer Imaging 17: 26, 2017.

9. Kerbauy MN, Pasqualin DDC, Smid J, Iquizli R, Kerbauy LN Nitrini R, Ribas GC, Neder L and Hamerschlak N: Diffuse large B-cell lymphoma of the central nervous system presenting as 'lymphomatosis cerebri' and dementia in elderly man: Case report and review of the literature. Medicine (Baltimore) 98: e14367, 2019.

10. Chen YF, Qiu QD, Wu H, Liu XM, Wu MM and Yan ZH: Imaging and pathological study of primary central nervous system lymphoma in special sites. Chin J Neurol 53: 700-705, 2020.

11. Izquierdo C, Velasco R, Vidal N, Sánchez JJ, Argyriou AA, Besora S, Graus F and Bruna J: Lymphomatosis cerebri: A rare form of primary central nervous system lymphoma. Analysis of 7 cases and systematic review of the literature. Neuro Oncol 18: 707-715, 2016.

12. Mora P, Majós C, Castañer S, Sánchez JJ, Gabarrós A, Muntané A, Aguilera $\mathrm{C}$ and Arús C: (1)H-MRS is useful to reinforce the suspicion of primary central nervous system lymphoma prior to surgery. Eur Radiol 24: 2895-2905, 2014.

13. Imataki O, Uchida S, Yokokura S, Uemura M and Kadowaki N: Central nervous system peripheral $\mathrm{T}$ cell lymphoma manifesting as lymphomatosis cerebri that was misdiagnosed as neuro-behçet's disease: A case report. Case Rep Oncol 11: 806-813, 2018.

14. Imperiale D, Taraglio S, Atzori C and Testi R: Diffuse leukoencephalopathy due to lymphomatosis cerebri: A clinicopathological report. Neurol Sci 36: 1071-1073, 2015.

15. Sato H, Takahashi Y, Wada M, Shiono Y, Suzuki I, Kohno K, Kato Y, Kawanami T, Sakurada K, Kayama T and Kato T: Lymphomatosis cerebri with intramedullary spinal cord involvement. Intern Med 52: 2561-2565, 2013.

16. Murakami T, Yoshida K, Segawa M, Yoshihara A, Hoshi A, Nakamura K, Ichikawa M, Suzuki O, Yokoyama Y, Toyoshima Y, et al: A case of lymphomatosis cerebri mimicking inflammatory diseases. BMC Neurol 16: 128, 2016.

17. Giannini C, Dogan A and Salomão DR: CNS lymphoma: A practical diagnostic approach. J Neuropathol Exp Neurol 73: 478-494, 2014.

\footnotetext{
This work is licensed under a Creative Commons Attribution-NonCommercial-NoDerivatives 4.0 International (CC BY-NC-ND 4.0) License.
} 